\title{
LES ANTAGONISMES DE CLASSES
}

\author{
DANS LA LITTÉRATURE SOCYALE FRANÇAISE \\ DE SAINT-SIMON A I 848
}

Soit qu'on l'en blâme, soit qu'on l'en loue, on rapporte souvent au Marxisme la paternité de l'idée des oppositions de classes dans la société industrielle. Nous voudrions montrer ici qu'il y a là une vue excessivement schématique et que, s'il est vrai que c'est le Marxisme qui a donné à cette idée sa portée sociale, sa portée de propagande surtout, la notion des "antagonismes" immanents à la société contemporaine s'est formée bien avant les premiers manifestes du Marxisme. Nous utiliserons pour cela la littérature sociale française en prenant pour point de départ les oeuvres de Saint-Simon et en nous bornant à suivre l'évolution de cette idée dans la lignée de quelques auteurs qui se rattachent à son influence, jusqu'en i 848 .

Ce point de départ pris dans l'oeuvre de Saint-Simon n'est pas entièrement arbitraire. Comme il le dit lui-même ${ }^{1}$, Henri de SaintSimon est le témoin d'une "époque de transition". Certes, "la guerre du capital et du travail n'est pas encore déclarée de son vivant; l'emploi des machines, le développement de la grande industrie, l'accumulation des ouvriers dans de gigantesques fabriques n'ont pas encore produit leurs effets". ${ }^{2}$ Il ne faudrait pas méconnaître toutefois les profondes transformations qui, en France, ont accompagné la Révolution et l'Empire. Les progrès de la technique, des machines à tisser et à filer notamment, les inventions de Jacquard et de Philippe de Girard, les initiatives de Richard Lenoir, bientôt après, les premières machines à vapeur, la nécessité aussi, par suite du blocus continental, de produire en France ce qui venait jusque-là de l'étranger,

1 Catéchisme des industriels, Ier cahier ( 1823 ), dans les Euvres de Saint-Simon et d'Enfantin, t. 28 (VIII), p. 4 I. - Nous citons généralement d'après cette édition (Paris, Dentu, $1869-1876$; puis E. Leroux, $1877^{-1878)}$ en donnant d'abord la tomaison générale, puis entre parenthèses et en chiffres romains, la tomaison propre aux oeuvres de Saint-Simon lui-même.

2 Georges WeIll, Saint-Simon et son oeuvre, Paris, Perrin, 1894, p. 238. 
les conquêtes impériales qui ouvraient au commerce français, temporairement au moins, de nouveaux débouchés, tout cela avait imprimé à la production française une impulsion nouvelle. Les crises économiques qui s'annoncent dès 1810 , n'en furent que plus brutales et, en dépit de la reprise de l'essor industriel à partir de i 8 I 5 , elles continuent encore pendant la Restauration: en 1817 , on dut allouer aux boulangers parisiens une subvention de 24 millions de francs pour faire baisser le prix du pain, qui avait augmenté de façon exorbitante.

Or Saint-Simon a vécu toutes ces vicissitudes. D'origine noble, comme on sait, ruiné par la Révolution, puis enrichi par ses spéculations, mais presque aussitôt ruiné à nouveau, parfois réduit à la misère, il éprouva tous les contre-coups des événements politiques et des transformations économiques. Doué, sinon d'une pensée très équilibrée, du moins d'un don d'intuition et d'anticipation incontestables, il traduit dans ses idées la période tourmentée qu'il a traversée.

Dès I 803 , dans ses Lettres d'un habitant de Genève, il déclare apercevoir dans la société, les savants et les artistes mis à part, deux grandes "classes": celle des propriétaires et celle des non-propriétaires, la première étant en comparaison de la seconde très peu nombreuse, et il tient aux membres de cette dernière le petit discours que voici:

"Vous dites: nous sommes dix fois, vingt fois, cent fois plus nombreux que les propriétaires, et cependant les propriétaires exercent sur nous une domination bien plus grande que celle que nous exerçons sur eux. Je conçois, mes amis, que vous soyez très contrariés; mais remarquez que les propriétaires, quoique inférieurs en nombre, possèdent plus de lumières que vous et que, pour le bien général, la domination doit être répartie dans la proportion des lumières." 1

Mais c'est surtout à partir de i 8 is que la pensée de Saint-Simon se précise. Il comprend alors l'importance des transformations économiques dont il est témoin et il invente le terme d'industrialisme pour exprimer son idéal social et le substituer à celui de libéralisme. L'un des avantages de ce terme est, dit-il, qu'il indique à la fois le but: "celui de donner pour base à l'organisation sociale l'intérêt de la majorité", et les moyens, qui sont de "confier aux industriels les plus importants l'administration de la fortune publique"."2

C'est qu'en effet, malgré les crises dont il a été et est encore témoin, Saint-Simon ne partage nullement le pessimisme de certains économistes tels que Sismondi, qui écrira en 18 I9: "Les économistes ne cessent d'encourager les nations à produire, à inventer de nouvelles

1 Édition Olinde Rodrigues, Paris, Capelle, r 841, 2me partie, p. 40.

${ }^{2}$ Le Libéralisme et l'Industrialisme, dans l'éd. Olinde Rodrigues, Ière partie, p. 205 et suiv. 
machines, ils signalent les oisifs à l'indignation publique, ... ils voudraient que chacun fût ouvrier, que chacun travaillât pour vivre. Mais l'homme ne se fatigue que pour se reposer ensuite." 1 Bien au contraire, Saint-Simon s'enthousiasme pour les nouvelles formes de production:

"La société tout entière repose sur l'industrie. L'industrie est la seule garantie de son existence, la source unique de toutes les richesses et de toutes les prospérités. L'état de choses le plus favorable à l'industrie est donc, par cela seul, le plus favorable à la société. Voilà tout à la fois et le point de départ et le but de tous nos efforts". ${ }^{2}$

Il chante les bienfaits du travail. Dès y 808 , dans son Introduction aux travaux scientifiques du XIX ${ }^{\circ}$ siècle, il avait écrit: "L'homme le plus heureux est celui qui travaille." En décembre I823, dans le premier cahier du Catéchisme des industriels ${ }^{3}$, il répètera: "Le travail est la source de toutes les vertus; les travaux les plus utiles sont ceux qui doivent être les plus considérés; ainsi la morale divine et la morale humaine appelent également la classe industrielle à jouer le premier rôle dans la société". Ce thème revient comme un leit-motiv dans toute son oeuvre; le problème est, pour lui, de savoir "si la nation doit être organisée dans l'intérêt des militaires, des riches oisifs et des fonctionnaires publics, ou bien dans celui des producteurs". 4

La "classe industrielle", il y revient sans cesse, constitue "les vingtquatre vingt-cinquièmes de la nation". ${ }^{5}$ Non seulement elle est la plus nombreuse et, à Paris, elle est "plus importante que toutes les autres classes réunies".6 Mais c'est "celle qui produit toutes les richesses" 7 - nous retrouverons cette formule aux approches de 1848 , mais avec un autre "son de cloche" - et ainsi elle est "la classe fondamentale, la classe nourricière de toute la société": certes, "les savants rendent des services très importants à la classe industrielle, mais ils reçoivent d'elle des services bien plus importants encore, ils en reçoivent l'existence" 8 - on sait que c'est sur ce point que portera le désaccord de Saint-

1 Nouveaux Principes d'Economic politique, t. I, p. 78-80.

2 Guvres, t. 18 (II), p. I3.

3 Guvres, t. 37 (VIII), p. 43.

4 Du Système industricl (1821), Ceuvres, t. 22 (VI), p. 95.

${ }^{5}$ Catéchisme des industriels, CEuvres, t. 37 (VIII), p. 13, p. 53, etc. - Dans le Systeme industriel, $2 \mathrm{me}$ partie, Euvres, t. 22 (VI), p. 187, il avait évalué la proportion à 29 millions $1 / 2$ sur 30 millions de Français.

6 Ibidem, p. 52.

7 Ibidem, p. I3, p. 62, etc. - Même formule, Systeme industricl, Guvres, t. 22 (VI), p. 79.

8 Ibidem, quatrième cahier, Euvres, t. 39 (X), p. 25. 
Simon avec Auguste Comte, celui-ci se refusant à subordonner la science théorique à la technique -. Or, dans l'organisation actuelle, la classe en question occupe, sans d'ailleurs qu'elle en ait conscience, une situation "tout à fait subalterne"; elle est "la dernière de toutes".'

La stratification sociale se présente donc, aux yeux de Saint-Simon, sous la forme suivante. Avant la Révolution, la nation comprenait trois classes: la noblesse, la bourgeoisie et les "industriels": "Les nobles gouvernaient, les bourgeois et les industriels les payaient." Aujourd'hui, il semble à première vue qu'il existe encore une "classe intermédiaire": celle qui, sous la Restauration, fournit la clientèle du parti libéral: "Le parti libéral a toujours et aura toujours pour directeurs les classes intermédiaires". ${ }^{2}$ Mais, en réalité, cette classe "intermédiaire" n'est autre que la bourgeoisie; or c'est la bourgeoisie (et non les industriels, qui sont "essentiellement pacifiques") qui a fait la Révolution et elle en a bénéficié: les privilèges de la noblesse ayant été anéantis, elle a eu accès au gouvernement et, si elle a jadis rendu des services aux industriels, "aujourd'hui la classe bourgeoise pèse avec la classe noble sur la classe industrielle" 3, de sorte que "la nation n'est plus partagée qu'en deux classes" et que "les industriels doivent payer les nobles et les bourgeois". ${ }^{4}$

Dans sa célèbre Parabole, parue en 18 I9 dans la première livraison de l'Organisateur ${ }^{5}$, Saint-Simon présente cette division de la société en deux classes sous une forme saisissante, - si saisissante qu'elle conduisit son auteur sur les bancs de la Cour d'assises, qui d'ailleurs l'acquitta -. Il y fait deux hypothèses: il suppose d'abord que la France perde, dans chaque spécialité, ses cinquante premiers savants (ses cinquante premiers physiciens, ses cinquante premiers chimistes, etc), ses cinquante premiers artistes (peintres, sculpteurs, etc), ses cinquante premiers ingénieurs, médecins, pharmaciens, etc, ses cinquante premiers banquiers, ses cinquante premiers chefs d'industrie dans les différents ordres de production (il n'énumère pas moins de 24 corps de métiers), ses deux cents premiers négociants et ses six cents premiers cultivateurs. Il imagine d'autre part que la même perte frappe les membres de la famille royale, les grands officiers de la Couronne, ministres d'Etat, préfets, juges, etc, et "les dix mille propriétaires les plus riches parmi ceux qui vivent noblement". Dans la première hypothèse, la nation deviendrait "un corps sans âme", toute sa vie

1 Ibidem, t. 37 (VIII), p. 4-6.

2 Ibidem, p. 52.

3 Ibidem, p. I I et 39.

4 Ibidem, p. 8.

5 On la trouvera soit dans les (Euvres, éd. citée, t. 20 (IV), p. 17-26, soit dans l'éd. Olinde Rodrigues, 2me partie, p. $7 \mathrm{I}-80$. Elle est aussi reproduite dans les Euvres choisies de C.-H. de Saint-Simon, Bruxelles, Fr. van Meenen, 1859 , t. II, p. 395-40I. 
serait paralysée. Dans la seconde au contraire, "cette perte affligerait certainement les Français parce qu'ils sont bons, ... mais cette perte des trente mille individus réputés les plus importants de l'Etat ne leur causerait du chagrin que sous un rapport purement sentimental, car il n'en résulterait aucun mal politique pour l'Etat", d'autant plus, ajoute, non sans humour, Saint-Simon, qu'il serait très facile de les remplacer.

Cette Parabole hardie illustre avec vigueur la théorie de SaintSimon: il n'y a dans la société que deux classes: ceux qui produisent, et donc qui sont utiles, et ceux qu'il appelle "les oisifs", qui vivent aux dépens des premiers. Deux ans plus tard, dans une lettre au Garde des Sceaux 1, il parlera avec une certaine âpreté de "la lutte qui doit finir par exister entre la masse entière des parasites d'un côté et la masse des producteurs de l'autre, pour décider si ceux-ci continueront à être la proie des premiers". Voilà qui semble annoncer le ton véhément de certaines formules revendicatrices que l'on entendra bientôt.

$\mathrm{Ne}$ nous y trompons pas cependant et demandons-nous ce que Saint-Simon entend exactement par ces deux classes des "producteurs" et des "oisifs" ou, comme il dit encore, des abeilles et des frelons. ${ }^{2}$

"Producteurs", "industriels", "parti national" opposé au "parti antinational" " 3 , tous ces termes sont pour lui à peu près synonymes. Tout au plus admet-il dans le "parti national", outre les "industriels", les artistes et les savants, du moins ceux de ces derniers qui s'occupent de "sciences positives", et ce qualificatif de positif a déjà pour lui la pluralité de sens qu'il aura pour Auguste Comte: il signifie à la fois constructeur (par opposition à négatif ou destructeur), fondé sur les faits et aussi, et peut-être surtout, utile. Ainsi que le remarque Fr. Muckle 4, il semble que ce soit d'après le "degré d'utilité" qu'elles présentent pour la nation que Saint-Simon ait conçu la hiérarchie des couches sociales. Les seuls travaux utiles à la société, écrit-il dans l'Organisateur en $18199^{5}$, sont "les sciences, les beaux-arts et les arts et métiers". Par suite, méritent seuls le nom de "travailleurs" les savants, les artistes et "les artisans". En dehors de là, "on ne trouve que les parasites et les dominateurs". Quant à ce qu'il entend par "artisans", une note de la Parabole le précise de façon bien curieuse: "On ne désigne ordinairement par artisans que les simples ouvriers; pour éviter les circonlocutions, nous entendons par cette expression tous ceux qui s'occupent de produits matériels, savoir: les cultivateurs,

1 CEuvres, t. 22 (VI), p. 258.

2 Sur la querelle des abeilles et des frelons ou sur la situation respective des producteurs et des consommateurs non producteurs, avril I8 8 9, GEuvres, t. I9 (III), p. 2 I I et suiv.

3 Guvres, t. 19(III), p. 195.

4 Henri de Saint-Simon, die Persönlichkeit und ihr Werk, Jena, G. Fischer, 1908, p. 193.

5 Euvres, t. 20(IV), p. 191-I92. 
les fabricants, les commerçants, les banquiers et tous les commis ou ouvriers qu'ils emploient". ${ }^{1}$

Le terme d'industriels est pris par lui en un sens tout aussi large. En I82I, il adresse une lettre "à MM. les cultivateurs, fabricants, négociants, banquiers et autres industriels". 2 Le Catéchisme des Industriels s'ouvre par cette définition: "Un industriel est un homme qui travaille à produire ou à mettre à la portée des différents membres de la société un ou plusieurs moyens matériels de satisfaire leurs besoins ou leurs goûts physiques" 3 , et, de nouveau, il divise "la classe des industriels" en "trois grandes classes" qui sont les cultivateurs, les fabricants et les négociants (cette fois, les banquiers ne font pas l'objet d'une mention spéciale). Il avait classé auparavant ${ }^{4}$ parmi les "oisifs", - ceux qui forment le "parti anti-national" - "les propriétaires terriens qui vivent noblement", c'est-à-dire, précise-t-il, "sans rien faire", tous ceux "qui consomment et ne produisent point", et aussi "ceux dont les travaux ne sont point utiles à la société et ne servent point aux producteurs", voire "ceux qui professent des principes politiques dont les applications nuisent à la production", tels "ceux des prêtres qui font consister la morale dans la crédulité aveugle aux décisions du pape et du clergé, ... les juges qui soutiennent l'arbitraire, les militaires qui lui prêtent leur appui", etc., ou encore, comme il lui arrive de dire, "les sabreurs et les tonsurés".

On a certainement remarqué que, dans ces diverses classifications, Saint-Simon ne réserve aucune place à part pour les ouvriers ou, d'une façon générale, les salariés. Il les englobe, avec les chefs d'entreprises, sous le vocable d'artisans ou d'industriels. Pourtant, on ne peut s'empêcher de penser que, lorsqu'il parle de ceux-ci comme d'une classe injustement infériorisée, c'est au moins en partie aux ouvriers qu'il pense. C'est à eux, de toute évidence, que s'applique la formule qu'il lancera bientôt dans le Nouveau Christianisme et qui deviendra le mot d'ordre de "l'école saint-simonienne": "Améliorer la situation physique et morale de la classe la plus nombreuse et la plus pauvre." Et de qui, si ce n'est des salariés, pourrait-il être question lorsqu'il déclare que son but est d'améliorer le sort de cette classe "qui n'a pas d'autres moyens d'existence que le travail de ses bras", et dont, ajoutet-il, les gouvernements, à l'étranger comme en France, ne s'occupent que pour "la maintenir dans l'obéissance la plus passive" 5 ?

1 Ibidem, p. I9, note.

2 CEuvres, t. 22 (VI), p. 78.

3 CEuvres, t. 27 (VIII), p. 3.

4 Cuvres, t. 19(III), p. 195-205.

5 Euvres, t. 22 (VI), p. 8I. - Un détail peut préciser le sens que Saint-Simon donnait au mot industriel: Rouget de Lisle avait composé pour lui un Chant des Industriels; il s'empressa de le faire apprendre aux ouvriers de l'un de ses commanditaires, le manufacturier Ternaux, ... et fut d'ailleurs émerveillé du résultat. 
Et pourtant, si la transformation sociale qu'il préconise, doit s'accomplir en grande partie pour le peuple, Saint-Simon n'imagine pas un instant qu'elle puisse s'accomplir par le peuple. Nous sommes encore bien loin, avec lui, de la formule marxiste: "L'émancipation des travailleurs sera l'oeuvre des travailleurs eux-mêmes", disons même: de toute formule démocratique. Il se défie des idées libérales, il proclame qu'il faut mettre un terme à la Révolution, il répète sans cesse que "les chefs des travaux industriels sont les protecteurs nés de la classe ouvrière", et il insiste:

"Tant que les manufacturiers feront bande à part avec les ouvriers, tant qu'ils ne tiendront pas en politique un langage qui pourra être entendu par eux, l'opinion de cette classe très nombreuse et encore très ignorante ne se trouvera point guidée par ses chefs naturels, elle pourra toujours se laisser séduire par les intrigants qui voudront faire des révolutions pour s'emparer du pouvoir" 1.

Voilà bien, en effet, ce qui inquiète Saint-Simon; et on le comprend un peu: la Révolution, - dont il avait eu à souffrir - n'était pas encore tellement loin! Une société, dit-il ${ }^{2}$, "dans laquelle deux principes antagonistes se trouvent admis", risque d'aboutir au désordre. Si l'état de choses actuel se prolonge, "la tranquillité publique sera de plus en plus menacée". Or "les moyens violents sont bons pour détruire, mais ils ne sont bons que pour cela".

Aussi bien, n'est-ce pas aux ouvriers qu'il s'adresse le plus souvent et, dans le Nouveau Christianisme ${ }^{3}$, il expliquera pourquoi: c'était, dira$\mathrm{t}$-il, "pour que l'émission de la nouvelle doctrine ne portât point la classe pauvre à des actes de violence contre les riches et contre les gouvernements". Au contraire, "les chefs des travaux industriels sont de tous les citoyens les plus intéressés à la tranquillité". 4 Et, par suite, c'est à eux, c'est aux "industriels les plus importants", aux "principaux industriels", aux "chefs des différents travaux" - et, comme pour ne laisser subsister aucune équivoque, il précise qu'il entend par là "tous les industriels qui ne sont pas purement ouvriers, c'est-à-dire exécutants, et qui prennent une part plus ou moins grande à la direction des travaux" - c'est à ceux-là que vont presque toujours ses exhortations et ses adjurations: car, explique-t-il, c'est en eux "que se trouve exclusivement la puissance d'agir sur le peuple, parce que c'est à eux que le peuple est habituellement subordonné dans ses relations jour-

1 Edition Olinde Rodrigues, tère partie, p. 221.

2 Euvres, t. 37 (VIII), p. 9 et p. 34 .

3 Edition Olinde Rodrigues, 2 me partie, p. 174.

4 Euvres, t. 22 (VI), p. 245. 
nalières".' La seule chose qu'il leur reproche, c'est qu'ils ' $n$ 'éprouvent point le sentiment de la supériorité de leur classe". ${ }^{2}$

Certes, Saint-Simon concède parfois que, dans le système "industriel", les ouvriers devront être associés à leurs employeurs plutôt que proprement subordonnés. Son "disciple" Auguste Comte l'avait déjà écrit dans l'Organisateur ${ }^{3}$, mais - détail digne de remarque - en appliquant cette réflexion à l'état actuel: dans le système militaire, il y avait commandement; dans le système industriel, il n'y a plus que direction: "Dans le premier cas, le peuple était sujet; dans le second, il est sociétaire": "tous collaborateurs, tous associés, depuis le plus simple manouvrier jusqu'au manufacturier le plus opulent". Plus tard, dans son étude sur l'Organisation sociale, Saint-Simon reprend à peu près la même formule, mais en l'appliquant à l'avenir: "La classe des prolétaires étant, dit-il, aussi avancée en civilisation que celle des propriétaires, la loi doit les classer comme sociétaires".4

Il n'en reste pas moins que cette association, telle qu'il la conçoit, doit être essentiellement hiérarchique. Il déclare sans doute que "le système industriel est fondé sur le principe de l'égalité parfaite". Mais c'est seulement en tant qu'il s'oppose au système féodal et aux privilèges héréditaires de la noblesse. ${ }^{5}$ D'après lui, "la véritable égalité" consiste en ce que "chacun retire de la société des bénéfices exactement proportionnés à sa mise sociale, c'est-à-dire à sa capacité positive, à l'emploi utile qu'il fait de ses moyens", et Saint-Simon prend bien soin de préciser: "parmi lesquels il faut comprendre, bien entendu, ses capitaux". ${ }^{6}$ Loin de s'en prendre aux riches indistinctement, il pense au contraire que "la richesse est, en général, une preuve de capacité chez les industriels, même dans le cas où ils ont hérité de la fortune qu'ils possèdent", tandis que, dans les autres classes, il est toujours vraisemblable que les riches sont inférieurs en capacité à ceux qui ont reçu une éducation égale à la leur. ${ }^{7} \mathrm{Il}$ y a, dit-il, deux espèces de fortunes: celles "qui résultent d'opérations hasardeuses et d'agiotages" et celles "qui résultent de découvertes importantes dans les sciences, dans les beauxarts et dans les arts-et-métiers": ces dernières "ne sont jamais acquises aux dépens des individus ni de la nation et elles tournent constamment au profit de la masse entière de la société". ${ }^{8}$ Aussi, lorsqu'il envisage des modifications possibles à la structure de la propriété, Saint-

\footnotetext{
1 Ibidem, p. 2 I 8.

2 Guvres, t. 37 (VIII), p. IO-I I, p. 54-55, etc.

3 (Euvres, t. 20 (IV), p. I 50.

4 CEuvres, t. $39(\mathrm{X})$, p. I25.

5 Euvres, t. 37 (VIII), p. 6r.

6 CEuvres, t. 22 (VI), p. I7, note.

7 Euvres, t. 2 I (V), p. 49, note.

8 Euvres, t. 2o(IV), p. 209.
} 
Simon se borne-t-il à affirmer que "le droit individuel de propriété ne peut être fondé que sur l'utilité commune" 1 sans préciser les applications qu'il entend donner à ce principe. - Ce respect de la richesse, il veut que les ouvriers eux-mêmes s'en inspirent et, dans une des rares lettres qu'il leur adresse, il leur conseille de tenir à leurs employeurs le langage suivant: "Vous êtes riches, et nous sommes pauvres; vous travaillez de la tête, et nous des bras; il résulte de ces deux différences fondamentales que nous sommes et que nous devons être vos subordonnés". 2

Les idées de Saint-Simon reflètent à la fois son expérience personnelle et l'état de la société dans laquelle il vivait. Comme il a été dit au début, celle-ci n'était pas encore tourmentée par les oppositions qui allaient se faire jour un peu plus tard, et les conflits sociaux aigus étaient encore relativement rares. C'est ce qui explique que Saint-Simon, bien qu'ayant aperçu quelques-uns des problèmes qui commençaient seulement à se poser, ne nous livre qu'une notion encore très vague et très incertaine de la "classe" sociale. On a certainement remarqué, dans les textes que nous avons cités, l'emploi très variable et très flottant qu'il fait de ce terme. Tantôt il parle de "la classe industrielle" au singulier, en y incluant d'ailleurs, comme nous l'avons souligné, chefs d'entreprises et ouvriers; tantôt il y distingue plusieurs groupes qu'il appelle également des "classes": cultivateurs, fabricants, négociants; tantôt il risque l'expression: "classe des prolétaires"; parfois il dit: "classe riche" et "classe pauvre". Il lui arrive de substituer le terme "parti" au terme "classe" et de parler du "parti industriel", qui est pour lui le seul "parti national". On a vu enfin qu'à ses yeux les banquiers sont des "industriels", voire des "artisans",... tout comme les ouvriers! Ces incertitudes de langage ne sont pas seulement l'expression de l'incoordination de pensée de Saint-Simon. Elles traduisent l'idée confuse qu'en cette période de transition, pouvait se faire de la stratification sociale un esprit perspicace certes et qui a compris que l'avènement de la grande industrie posait des problèmes nouveaux, mais qui, ainsi que l'a dit Muckle ${ }^{3}$, n'a pu prendre qu'une vague conscience "des oppositions qui divisaient en deux camps ceux qu'il appelle les industriels", de sorte que "le problème de l'exploitation capitaliste en tant qu'il diffère de celui de l'exploitation féodale lui est encore demeuré presque entièrement fermé".

On sait que les disciples de Saint-Simon, tout en se réclamant de leur maître comme du révélateur d'une foi nouvelle, ont profondément

${ }_{1}$ CEuvres, t. I9 (III), p. 89-9o.

2 Lettre à MM. les Ouvriers, extrait du Système industricl, 2me partic (Paris, Bibl.

Nationale, R 26, 2), p. 21 3-214.

${ }^{3}$ H. de Saint-Simon, die Persönlichkeit und ihr Werk, p. 205. 
modifié sa doctrine. C'est qu'en effet, dans les dernières années de la vie du maître, les circonstances ont changé: en i 824, la France connaît la première crise générale, comparable à celles que connaît déjà l'Angleterre; en 1825 , le Journal des Débats ${ }^{1}$, parlant d'une grève accompagnée de violences dans une fabrique de Rouen, la qualifie de "révolte jusqu'alors sans exemple"; et, lorsque, dans une série de conférences tenues depuis décembre i 828 jusqu'au début de 1830 , Bazard expose la Doctrine de l'école, la Révolution de Juillet est toute proche. On a remarqué d'ailleurs ${ }^{2}$ que, dans cette Exposition de la Doctrine, Bazard "a les yeux fixés sur l'avenir beaucoup plus que sur le passé", tandis que Saint-Simon semblait encore hanté par la pensée de l'ordre d'Ancien Régime opposé à l'ordre "industriel" naissant. D'autre part, tandis que le maître s'adressait surtout, nous l'avons dit, aux chefs d'industrie, ses disciples s'adressent d'abord aux intellectuels - on sait qu'ils eurent parmi eux toute une cohorte de polytechniciens, - puis, à partir de i 830 , bien qu'assez timidement, au peuple lui-même. Quoi qu'il en soit, cette Exposition de la Doctrine de Saint-Simon ${ }^{3}$ constitue un document capital dans l'histoire des idées sociales, en particulier au point de vue qui nous occupe ici, et c'est à elle que nous nous réfèrerons le plus souvent.

Nous y voyons d'abord apparaître des formules nouvelles qui marquent un changement d'orientation sensible. Le mot même d'antagonisme, rare chez Saint-Simon (nous ne l'avons relevé qu'une fois chez lui, dans un texte cité ci-dessus), devient courant dans la Doctrine: il figure dans le titre de la 4me séance où il est opposé à "l'association universelle". A vrai dire, les saint-simoniens lui donnent encore un sens assez large: tantôt il s'agit de l'antagonisme entre le pouvoir spirituel et le pouvoir temporel ${ }^{4}$, tantôt c'est la guerre qui est signalée comme "la manifestation la plus vive de l'antagonisme" 5. Mais le terme sert aussi parfois à désigner ce qu'on appellera bientôt la "lutte de classes". C'est ainsi qu'il est dit, toujours dans cette 4 me séance, que l'antagonisme a régné jusque-là non seulement entre les peuples et les nations, non seulement entre cités ou provinces faisant partie d'une même nation, mais aussi "dans l'intérieur de la cité entre les différentes classes d'hommes qui la composent" et, à ce propos, l'auteur ose cette remarque, vraiment curieuse dans le contexte où elle se situe, - car elle semble annoncer certains jugements de valeur que ne désavouerait pas,

1 I I août 1825 , cité par G. WEILL, op. cit., p. 328, note.

2 Emile Durkherm, Le Socialisme, Alcan, 1928 (posthume), p. 304.

3 Nous citons d'après l'éd. de la Librairie nouvelle, Paris, 1854 , en ajoutant, pour la première année (1 828-1829) les références à l'éd. Bouglé et Halévy (que nous désignons par le sigle B.H.), Marcel Rivière, Paris, 1924.

4 Ière séance, P. 43 ; B.H., p. I 22.

54 me séance, p. 104; B.H., p. 2 I 2. 
croyons-nous, un marxiste de la plus stricte obédience: "Dans ces derniers cas [la lutte entre classes], la lutte n'a pas le même aspect dans tous les partis qui s'y trouvent engagés: chez l'esclave, chez le plébéien, elle a le caractère progressif, car elle a pour objet l'affranchissement du travail pacifique; chez le patricien, chez le maître au contraire, sa tendance est stationnaire ou rétrograde, car elle a pour objet le maintien des intérêts de la conquête, la prolongation du règne de la violence". ${ }^{1}$ Rapprochons-en cette autre réflexion qui suit presque aussitôt: "On peut dire que l'antagonisme, en préparant les voies d'une association plus large, en hâtant le jour de l'association universelle, se dévorait peu à peu lui-même". 22 Comment ne pas remarquer ici, avec Bouglé et Halévy, que "l'expression fait penser à la dialectique hégélienne dont s'inspirera la philosophie marxiste de l'histoire? L'antagonisme qui se dévore lui-même, c'est la négation qui se nie". Toute la fin de la 4me séance est d'ailleurs consacrée à retracer l'évolution de l'antagonisme et de "l'exploitation de l'homme par l'homme" qui en résulte, et à marquer que "la classe des travailleurs de l'ordre matériel n'est que le prolongement de celle des esclaves et des serfs". ${ }^{3}$ On observera ici encore, avec Gide et Rist dans leur Histoire des doctrines économiques 4 , que cet appel à "l'argument historique" prélude aux développements des théoriciens socialistes, marxistes surtout, du $\mathrm{XIX}^{\circ}$ siècle.

Quant à cette expression d'exploitation de l'homme par l'homme dont on sait quelle sera aussi la fortune, elle est également nouvelle. Certes, Saint-Simon avait indiqué à plusieurs reprises qu'à la domination sur les hommes qui caractérise le régime gouvernemental, il faut substituer l'exploitation du globe et l'action sur la nature avec un régime simplement administratif. ${ }^{5}$ Mais il n'avait jamais, à notre connaissance, appliqué le terme d'exploitation aux rapports humains. Ses disciples opposent au contraire très explicitement "l'exploitation de l'homme par l'homme" à "l'exploitation du globe par l'industrie" qui doit être le régime de l'avenir. ${ }^{6}$ Toute la 6 me séance de l'Exposition est consacrée à l'histoire des différentes phases de cette "exploitation de l'homme par l'homme" depuis la relation maitre et esclave, en passant par celle entre patricien et plébéien, puis par celle entre seigneur et serf, jusqu'à celle entre oisif et travailleur. Elle est, à leurs yeux, "le phénomène le plus caractéristique du passé" et, sans doute, elle va en

\footnotetext{
1 Ibidem, p. 102, note; B.H., p. 210 , note.

2 Ibidem, p. I03; B.H., p. 2 I I et note.

3 Ibidem, p. 105-108; B.H., p. 21 4-218.

- Ière éd., Larose et Tenin, Paris, I 909, p. 255.

3 Notamment Catéchisme des Industriels, Euvres, t. 37 (VIII), p. 87.

6 Introd., p. I I B.H., p. 88 ; - 2 me séance, p. 70; B.H., p. 162.
} 
décroissant, elle n'existe plus que "sous des formes tellement adoucies et détournées" qu'elle est parfois difficile à apercevoir. Elle subsiste cependant: elle "se continue à un très haut degré dans les relations des propriétaires et des travailleurs, des maîtres et des salariés". Il suffit, ajoutent-ils, “de jeter un coup d'oeil sur ce qui se passe autour de nous pour reconnaître que l'ouvrier, sauf l'intensité, est exploité matériellement, intellectuellement et moralement comme l'était autrefois l'esclave": car l'ouvrier, "pressé par l'état de misère auquel il est réduit", n'a plus ni le temps ni même le désir de développer sa vie intellectuelle et affective. ${ }^{1}$ Le christianisme n'a supprimé que l'esclavage proprement dit, mais "il n'a pas détruit l'exploitation de l'homme par l'homme... Cette exploitation pèse encore aujourd'hui avec une grande intensité, dans toutes les sociétés européennes, sur l'immense majorité de la population: partout cette majorité est vouée à la misère, à l'abrutissement, à la dépravation; partout c'est son abaissement qui fait les frais des jouissances des classes privilégiées". ${ }^{2}$ Dans ses articles du Globe (183I), Enfantin insistera davantage encore sur "cette classification barbare" entre oisifs et travailleurs ${ }^{3}$ "qui assigne à une classe une large part de jouissances sans travail et au plus grand nombre un pesant fardeau de labeur", de sorte qu'il y a nécessairement "lutte entre eux" et que se perpétue "la guerre dans l'Etat, dans la cité et jusque dans le foyer domestique". Comme le remarquent Bouglé et Halévy, les saint-simoniens sont ici tout près de la formule fameuse: "L'histoire du genre humain, c'est l'histoire de la lutte des classes"; nous trouvons déjà chez eux "tout le langage qui sera bientôt celui des socialistes révolutionnaires".4

Socialistes, les saint-simoniens le sont en effet. Leur maitre, même s'il avait, comme nous l'avons indiqué, entrevu comme nécessaire des transformations dans le régime de la propriété, était demeuré plus près d'une sorte de technocratie que du socialisme. Chez ses disciples, la critique de la propriété est incomparablement plus nette - et plus audacieuse. - Ce qu'ils incriminent, c'est "la propriété par droit de naissance, et non par droit de capacité: c'est l'héritage" ${ }^{5}$ et, en I 830 , dans une lettre au président de la Chambre des Députés, ils ne craindront pas de réclamer la suppresion de ce dernier et demanderont que "tous les instruments du travail, les terres et les capitaux soient réunis en un fonds social" ", c'est-à-dire une organisation collectiviste de la

1 Gme séance, p. 122-125; B.H., p. 236-240.

2 me année, Ière séance, p. 337.

3 Articles des 7 et 14 mars 1831 , teproduits dans la brochure Economie politique ct politique, Paris, zme éd., mai 1832, p. 6 r et suiv.

B.H., préface, p. 66.

¿ 8 me séance, p. I59; B.H., p. 287.

- Cité par Gide et Rist, op. cit., p. 254. 
propriété. Et sans doute, s'ils condamnent ainsi la propriété privée des instruments de production, c'est, en grande partie, parce qu'elle leur paraît conduire à "l'anarchie" de la production: ils ont conservé de leur maittre le souci de "l'organisation", et la propriété répartie selon les hasards de la naissance échoit souvent, pensent-ils, à des incapables. Mais leur hostilité à la propriété privée vient aussi de ce qu'elle n'est rien d'autre, à leurs yeux, que "le privilège de lever une prime sur le travail d'autrui" 1 ou, comme dit la pétition de I 830 , "le privilège de vivre du travail d'autrui". Voilà une formule qui n'est plus très éloignée des invectives futures d'un Proudhon contre le "vol" du propriétaire et la constante "erreur de compte" qui existe entre lui et le travailleur, ni même des impitoyables analyses de Marx concluant à l'existence d'une "plus-value" et d'un "surtravail" non payé à l'ouvrier. $^{2}$

Aussi les termes de "classe ouvrière" ${ }^{3}$ ou de "classe prolétaire" sontils chez les saint-simoniens d'un emploi beaucoup plus courant que chez leur maître. Les économistes eux-mêmes, déclarent-ils, ont reconnu dans la société "l'existence d'une classe de prolétaires" et constaté ainsi "l'hérédité de la misère". ${ }^{4}$ N'arriva-t-il pas un jour au "Père" Enfantin, à la suite de déceptions éprouvées dans sa propagande, de proclamer: "Les bourgeois et les docteurs se sont retirés de moi; nous sommes prolétaires" 5 ? Et c'est en effet à partir de ce moment (1830) que le mot bourgeois est employé dans le langage saint-simonien avec le sens péjoratif qu'il aura désormais dans le langage socialiste. "Le bourgeois, en tant que bourgeois, dira Enfantin dans le Globe, ne produit rien, n'enseigne rien et n'a d'autre soin que lui-même". Pour se maintenir dans cette situation, il faut "que la bourgeoisie ait à sa disposition toutes les ressources de la chicane et, à la rigueur, la force des baïonnettes". C'est pourquoi les administrateurs d'aujourd'hui, qu'ils soient préfets, officiers municipaux, etc, ne sont autre chose qu' "un instrument des oisifs, des bourgeois, des propriétaires". ${ }^{6}$ Encore une idée dont la propagande socialiste fera son profit: le gouvernement et la force publique au service de la classe dominante!

$16 \mathrm{mc}$ séance, p. I 29 ; B.H., p. 247.

2 Cf Georges Werll, L'Ecole saint-simonienne, Alcan, 1896, qui déclare que certain article du Producteur où Enfantin affirme que "les travailleurs paient certaines gens pour qu'ils se reposent", contient en germe le Capital de K. Marx (op. cit., p. 9-Io). Un peu plus loin (p. 63), il écrit que "plusieurs des idées essentielles contenues dans l'Exposition de Bazard ont passé dans les écrits de Rodbertus et même de Karl Marx".

${ }^{3}$ Cf. notamment deux articles de l'avocat Rouen sous ce titre dans le Producteur, tome III, p. 304, et tome IV, page $29^{2}$.

4 Gme séance, loc. cit.

5 Cité par Bouglé, Chez les Prophètes socialistes, Alcan, I9 I 8, p. II.

${ }_{6}$ Articles des $2 \mathrm{r}$ mars et 25 avril I 83 I : cf. Economie politique et Politique, p. 98-99 et 145 . 
La Doctrine elle-même parlait déjà, en termes sévères, de cette "classe d'hommes" à qui la propriété fondée sur l'héritage "donne la faculté de vivre du travail des autres et dans une complète oisiveté", entretenant ainsi "l'exploitation d'une partie de la population, la plus utile, celle qui travaille et produit, au profit de celle qui ne sait que détruire". ${ }^{1}$ Même "l'industriel" - et l'on notera qu'ici le terme est pris au sens d'entrepreneur, d'employeur - ne trouve pas grâce aux yeux des intransigeants disciples: "L'industriel se soucie peu des intérêts de la société... Dans ceux qui suivent la même carrière, il ne voit que des ennemis, il les attend, il les épie, et c'est à les ruiner qu'il fait consister son bonheur et sa gloire". ${ }^{2}$ Un tel langage diffère sensiblement de celui du maître, qui considérait au contraire les industriels comme les dirigeants les plus qualifiés de la société! Les propagandistes de la "foi" saint-simonienne sont plus audacieux encore, et leurs "prédications" annoncent déjà certaines violences de langage ultérieures. C'est ainsi que l'avocat Laurent, le futur commissaire de l'Ardèche en 1848 , dans une "prédication" sur Le parti politique des travailleurs ${ }^{3}$, oppose "les hommes qui tiennent du hasard de la naissance le droit de vivre du labeur d'autrui" à ceux qui sont "condamnés par la naissance à entretenir péniblement par leurs travaux le luxe et le faste de quelques privilégiés héréditaires". Ces hommes, ajoute-t-il, se rencontrent dans tous les partis: "L'oisif patriote admet et chérit comme l'oisif royaliste le principe héréditaire, d'où dérive la misère des travailleurs royalistes et patriotes. Le bourgeois républicain comme le bourgeois monarchique tient à conserver scrupuleusement les lois faites par lui et pour lui contre le prolétaire". Nouvelle anticipation sur la critique socialiste ultérieure: les distinctions de classes sont plus importantes que les différences d'opinions politiques. Dans cette même conférence, Laurent met d'ailleurs en parallèle la question: Qu'est-ce que les prolétaires? avec la fameuse interrogation de Sieyès: Qu'est-ce que le tiers état? Et il y répond ainsi:

"En nous demandant aujourd'hui ce que sont les prolétaires, nous devons apprendre à notre tour à la France que cette classe dont l'oisive bourgeoisie ne parle le plus souvent qu'avec dédain et qu'elle traite comme une humble vassale, compose aussi l'immense majorité de la nation; qu'elle peuple les champs et les ateliers, donne son sang dans les batailles, cultive les sciences et les arts, fournit aux besoins de l'État et entretient, charme et embellit

\footnotetext{
17 me séance, p. 135; B.H., p. 255.

2 Ière séance, p. $54-55 ;$ B.H., p. 139.

${ }^{3}$ Euvres de Saint-Simon et d'Enfantin, t. 45, p. 98-126. - Sur Laurent de l'Ardèche, voir

G. WEILL, L'Ecole saint-simonienne, p. 51 .
} 
sous mille formes diverses l'existence des classes privilégiées qui l'exploitent et qui la méprisent". ${ }^{1}$

C'est donc sur les "esclaves" du monde moderne que comptent désormais les disciples de Saint-Simon pour propager leur nouvelle foi, de même, rappellent-ils, que la foi chrétienne s'est répandue par le monde en s'appuyant "principalement sur les esclaves". ${ }^{2}$ Laurent fait appel aux travailleurs de toutes opinions et de toutes croyances pour constituer un parti unique comprenant "les classes nombreuses qui produisent tout et ne possèdent rien". Bientôt d'ailleurs l'école institua un "Degré des Ouvriers" dont le directeur général, sous la surveillance de Claire Bazard, fut l'ingénieur Fournel: à l'assemblée d'octobre I 83 I, ce "degré" comptait près de deux mille ouvriers, tant "fidèles" que "catéchumènes" professant "la foi nouvelle". Plus tard, l'école devait fonder de petits journaux, telle la Ruche populaire ${ }^{3}$ dirigée par Jules Vinçard, spécialement adressés aux ouvriers et rédigés en partie par eux.

Toutefois, ainsi que l'a montré C. Bouglé dans son étude Saintsimoniens et Ouvriers ${ }^{4}$, la propagande dans les milieux ouvriers se heurta à certaines difficultés. Et on se l'explique aisément. En dépit de leurs formules parfois hardies, les disciples de Saint-Simon n'avaient pas complètement abandonné les points de vue du maître. Leur terminologie en porte encore la trace. C'est ainsi que, sauf quelques exceptions dont nous avons donné des exemples, ils emploient encore, en général, le mot industriels en englobant sous ce terme à la fois les ouvriers et leurs employeurs, les salariés et leurs "patrons". Dans la I 2 me séance de la 2 me année, intitulée précisément "L'industriel", il est question de "la classe industrielle" dans le sens de "la classe ouvrière", car il y est dit qu'elle est "constamment exploitée"; mais nous y lisons aussi que "les industriels ont pris part aux affaires publiques", qu'ils sont "entrés dans les assemblées" 5 , ce qui ne saurait guère s'appliquer, à cette époque, qu'aux chefs d'entreprises. Dans le Producteur, le saint-simonien Allier est obligé d'expliquer: "Quoi! dira-t-on, les industriels sont ceux qui travaillent: ce sont donc les travailleurs; le mot "industriel" était donc inutile... Ce n'est pas cela, répond-il: le travailleur est celui qui, de gré ou de force, pour son compte ou pour

1 CEuvres, t. 45, p. I Io. - On retrouvera cette idée chez les rédacteurs de l'Atelier (v. ci-dessous).

2 Souligné dans le texte: I 4 me séance, p. 282 ; B.H., p. 442.

3 La Ruche populaire (Paris, Bibl. Nationale: $\operatorname{Lc}^{2}$ 1463) vécut de décembre 1839 à décembre I 849. Mais elle avait cessé en 1843 d'être saint-simonienne: elle fut remplacée alors par l'Union (Bibl. Nat.: $\mathrm{Lc}^{2}$ 1556) qui parut de décembre 1843 à septembre 1846 .

4 Dans le recueil déjà cité Chez les Prophètes socialistes, p. I-50. Sur le Degré des Ouvriers, cf. aussi G. WEILI, L'Ecole saint-simonienne, p. 55 .

5 zme année, I 2 me séance, p. $46_{4}$ et 469. 
le compte d'autrui, remplit une tâche quelconque, dont le résultat peut, à sa connaissance, être fructueux ou stérile... L'industriel, au contraire, est celui qui travaille librement, en qualité de chef ou d'ouvrier, dans l'agriculture, les manufactures, le commerce ou la banque, à une chose qui, dans sa pensée, doit lui être utile directement, parce qu'il restera propriétaire du fruit de son travail".' A ce compte, combien d'ouvriers seraient des "industriels"? L'ambiguité que nous avions signalée chez Saint-Simon, se perpétue encore ici. Pas plus que leur maître, d'ailleurs, les saint-simoniens n'établissent une différence essentielle entre le salaire de l'ouvrier et le profit de l'entrepreneur: Enfantin lui-même écrit dans le Producteur:2 "Nous pensons que les profits diminuent et que les salaires augmentent ${ }^{3}$; mais nous comprenons dans ce mot de salaire le bénéfice de l'entrepreneur d'industrie, car nous considérons ce bénéfice comme le prix de son travail".

$\mathrm{Au}$ fond, les saint-simoniens n'avaient pas abandonné le principe hiérarchique posé par le maître. Une note de la Doctrine le dit expressément: "Dans la société industrielle ainsi conçue [telle qu'ils la préconisent], on voit partout un chef et des inférieurs, des patrons et des clients, des maîtres et des apprentis; partout autorité légitime, parce que le chef est le plus capable; partout obéissance libre, parce que le chef est aimé; ordre partout: aucun ouvrier ne manque de guide dans ce vaste atelier", etc. ${ }^{4}$ Et ailleurs: "Nous ne nous adressons pas aux passions populaires... C'est l'ordre que nous réclamons, c'est la hiérarchie la plus unitaire, la plus ferme, que nous appelons pour l'avenir". ${ }^{5}$ Ils constatent d'ailleurs qu'ils vont ainsi contre un courant d'opinion très puissant: "La hiérarchie est justement ce qu'il y a de plus difficile à admettre à une époque comme celle où nous vivons, où la victoire dont on s'applaudit le plus est précisément d'avoir brisé toute hiérachie et où la dignité de caractère consiste surtout à ne point reconnaître de supérieurs". ${ }^{6}$ Au reste, le principe de répartition sur lequel doit être fondée, d'aprés eux la société industrielle est loin d'être un principe égalitaire: "Chacun devra se trouver classé selon sa capacité, rétribué suivant ses oeuvres".?

Ces tendances "hiérarchiques" n'étaient pas sans soulever quelques résistances de la part des ouvriers sympathisants ou même adhérents à l'école. Des membres du Degré des Ouvriers se plaignaient que, jusque dans les réunions saint-simoniennes, les rangs restaient marqués entre

1 Le Producteur, tome II, p. 572-573.

2 Tome I, p. 245.

3 L'idée est sans doute empruntée à l'économiste Ricardo.

47 me séance, p. 149, note; B.H., p. 272.

5 me séance, p. I 58 ; B.H., p. 286.

6 me année, I 3 me séance, p. 486.

7 Gme séance, ad finem, p. I 30 ; B.H., p. 248. 
eux et les bourgeois, et le bon Vinçard lui-même se regimbait contre l'esprit des dirigeants: "Ce sont, disait-il, des classeurs qui, de leur propre autorité, nous parqueront à tout jamais, ainsi que des moutons, dans un cercle d'assujettissement dont nous ne devrons jamais sortir!'1 Les chefs étaient d'ailleurs des intellectuels, qui souvent ne savaient pas parler aux ouvriers leur langage, et ceux-ci se sentaient "gênés, et comme humiliés, dans ces réunions où on les endoctrinait".

C. Bouglé, à qui nous empruntons cette indication, conclut cependant que le saint-simonisme a favorisé l'action de la classe ouvrière, non seulement en apportant à son vocabulaire certaines formules, comme "l'exploitation de l'homme par l'homme" qui en feront désormais partie intégrante - nous avons vu qu'ils lui ont apporté d'ailleurs, outre ces formules, certaines idées fondamentales, - mais aussi en "hâtant, comme malgré lui, au coeur des prolétaires qu'il s'efforça d'enrôler, la fermentation du sentiment ouvrier" et en aidant "sans le vouloir" à la naissance de ce qu'on appellera un peu plus tard la "conscience de classe" du prolétariat. Et pourtant les saint-simoniens "maintiennent que le peuple doit s'incliner devant ceux qui sont en effet ses maîtres naturels, c'est-à-dire ceux qui sont désignés non point par une hérédité aveugle, mais par des capacités vérifiées: l'obéissance, dans ces conditions, est la vertu primordiale". Sans doute, "ils veulent bien se dévouer tout entiers à la masse". Mais ils ne veulent pas "s'en remettre à elle: c'est pourquoi partout où ils aperçoivent germe ou résidu de quelque institution démocratique que ce soit, ils croient que tout est perdu". ${ }^{3}$ Viendra bientôt un temps où la question se posera de façon toute différente!

Parmi les disciples de l'école, il en est un, - trop oublié aujourd'hui, que nous mettrons à part, d'abord parce qu'il s'en est lui-même séparé, ensuite parce qu'il a apporté à la question qui nous occupe, une contribution vraiment nouvelle.

Il s'agit de P. J. B. Buchez, qui devait être en 1848 le premier président de l'Assemblée Nationale. ${ }^{4}$ Lorsque, vers la fin de I 828 , se constitua le Collège de l'école, Buchez fut un de ses members, et quand, le ıo décembre, Bazard commença ses conférences d'exposition de la doctrine, Buchez siégeait au bureau à côté de lui, d'Enfantin et d'Olinde Rodrigues. Il ne devait pas tarder cependant à quitter l'école, et cela pour deux raisons principales. ${ }^{5} \mathrm{La}$ première est qu'il n'acceptait

1 C. Bouglé, Chez les Prophètes socialistes, p. 43.

2 Ibidem, p. 47-48.

3 Ibidem, p. 39-40.

4 Cf. notre petit livre P. J. B. Buchez et les origines du socialisme chrétien, dans la collection du centenaire de la Révolution de 1848 , P.U.F., I 948.

5 Cf. notre ćtude Un schisme saint-simonien dans Hommes et Idéologies de i 840, Marcel Rivière, Paris, 1956. 
pas la transformation de l'école en secte religieuse et que, devenu chrétien, il répugnait au panthéisme sentimental dont Enfantin avait fait le dogme de la nouvelle église. La seconde touche de plus près à notre propos: ancien carbonaro, ayant pris part au complot de Belfort (I 82 I) sous la Restauration, Buchez était beaucoup plus près du peuple que les autres "dignitaires" de l'école et ne partageait pas leur culte de la "hiérarchie". Les saint-simoniens lui reprochaient d'être resté "l'homme critique" (entendez : révolutionnaire) d'autrefois. ${ }^{1}$ Dès 1830 , il s'était mis en rapport avec des ouvriers, avait sollicité les avis de "ces hommes en veste et en souliers ferrés" et avait même reçu d'eux des mémoires écrits. Dix ans plus tard, ce furent quelques-uns de ses disciples qui fondèrent l'un des premiers journaux ouvriers français, l'Atelier. On va voir que ces contacts avec le monde ouvrier donnent à la conception que se fait Buchez des classes sociales une allure toute différente de celle de la conception saint-simonienne.

Nous trouvons un premier témoignage des sentiments de Buchez sur cette question dans un brouillon de lettre qui fait partie de ses Manuscrits déposés à la Bibliothèque historique de la Ville de Paris ${ }^{2}$ et qui est destiné au saint-simonien Curie ${ }^{3}$ et daté du 30 août 1829 . Buchez y insiste surtout sur les conséquences morales de la division de la société en deux classes: "L'exploitation de l'homme par l'homme, écrit-il à son ami, engendre plusieurs genres de vices autant chez ceux qui exploitent que chez les malheureux qui sont exploités". Buchez examine d'abord les vices de "la classe maîtresse": "Les propriétaires des instruments de travail (terres et capitaux) doivent être divisés en deux classes: les uns sont absolument oisifs; les autres dirigent l'exploitation de leurs instruments." Ces derniers qui "représentent dans notre état moderne les barons du i ze siècle", ne sont certes pas désintéressés: "Ils font de leurs semblables des instruments de jouissance égoîstes; mais au moins ils jouent un rôle utile".

"Il n'en est pas ainsi de l'autre classe de propriétaires; ceux-ci, et ils sont peut-être supérieurs en nombre aux premiers, sont de purs oisifs; leur existence est inutile à la société. Ce sont ceux-là qui nous gouvernent, qui remplissent la chambre des pairs, encombrent la chambre des députés, se disputent le pouvoir de Ministre. Ce sont ceux-là qui veulent une société stationnaire... L'oisiveté, a-t-on dit, est la mère de tous les vices. Réfléchissez,

1 Voir, dans l'étude citée, les lettres de Claire Bazard.

2 Carton 5, dossier Projets d'organisation sociale, fol. 530.

3 Et non: Curée, comme l'imprime M. J. B. Duroselle, dans ses Débuts du Catholicisme social en France, P.U.F., 195I, p. 85 . Le nom de Curie se retrouve dans plusieurs publications saint-simoniennes, notamment Procês en police correctionelle, I vol., Io7 pages, 1832. 
mon ami, sur cet axiome: il est d'une vérité absolute. L'oisif devient forcément égoïste; car ses intérêts sont étrangers à ceux de la société active, c'est-à-dire du grand nombre..."

Buchez développe longuement ce thème. Puis il en vient à des considerations plus générales:

"Examinez un instant quels sentiments la séparation des hommes en deux classes, l'une riche, l'autre pauvre, jette dans la société. Les Maîtres méprisent les pauvres; ils spéculent sur eux comme sur de la matière morte, ne leur pardonnent rien et leur imposent tous les genres d'obligations. Les Pauvres haïssent les riches; le sentiment de la haine et de l'envie, voilà leur lot, s'ils ne sont point tout à fait abrutis. Autrement, soumis comme du bétail, ils n'ont point de pensée que celle de servir; alors, indifférents au vice comme à la vertu, ils sont descendus presqu'à ce rang d'inertie qu'on attribue à la pure matière; ils ont dépouillé tout ce qui constitue l'homme, la spontanéité".

Les périodiques de l'école buchézienne dont le premier, le Journal des Sciences morales et politiques, titre bientôt changé en celui de L'Européen, commença à paraître en décembre I 83 I, renferment aussi plusieurs articles sur la question. Ces articles marquent un durcissement, une accentuation de sévérité à l'égard de ceux que les saint-simoniens regardaient encore comme les "chefs naturels" des ouvriers. Un article de Buchez intitulé Moyen d'améliorer la condition des salariés des villes ${ }^{1}$ est révélateur à cet égard. Buchez constate d'abord une double tendance: "l'une des chefs d'industrie à baisser les salaires, l'autre des salariés à offrir leurs bras à meilleur marché". Et, à ce propos, il parle de ces "intermédiaires" qui font la liaison entre "ceux qui commandent les travaux" et les ouvriers, à savoir les entrepreneurs. Voici comment il les juge:

"Il ne faut pas croire que ces intermédiaires soient pour quelque chose dans la bonne exécution du travail qu'ils semblent diriger: ils ne s'occupent même pas ordinairement de cela; c'est un ouvrier, un chef d'atelier qui est chargé de mettre en oeuvre les plans tracés, de diviser et de réunir les diverses parties de l'opération nécessaires à leur achèvement. L'entrepreneur n'intervient là que comme capitaliste ou, en d'autres termes, comme soumissionnaire, comme possesseur d'un local qui sert d'atelier et fournisseur des matières premières; pour cela, outre les avantages

1 Journal des Sciences morales et politiques (Paris, Bibl. Nationale: R 6356-6357), 17 décembre 1831, p. 36 et suiv. 
qu'il peut tirer à l'aide d'un marché plus ou moins habilement fait, il prélève sur la valeur nette du travail de chacun des ouvriers qu'il emploie, une somme de trente sous à deux francs au moins par journée. Nous n'exagérons pas; nous avons beaucoup consulté pour acquérir la connaissance exacte de la moyenne de ce prélèvement; et il résulte de nos recherches que la prime payée par l'ouvrier à l'entrepreneur n'est jamais moindre de trente sous et s'élève quelquefois au double, au quadruple, au quintuple, si l'on consulte le montant de ce que paie le consommateur'.

Evidemment, Buchez ne nous dit pas par quels procédés il a pu calculer le montant de ce "prélèvement". Mais ce qui intéressant, c'est de voir se préciser cette notion même d'un prélèvement opéré par la classe des employeurs sur le travail des ouvriers, notion déjà indiquée par les saint-simoniens, mais qui jouera, nous l'avons dit, un rôle si important dans la critique socialiste ultérieure.

Buchez conclut: "Ces entrepreneurs sont de purs parasites, dont l'intervention sans utilité, ruineuse pour le producteur et l'acheteur, est cependant si chèrement payée que rarement ils manquent de faire une fortune grande ou médiocre". Il revient à la charge dans un article de l'Européen du 30 juin 1832 (p. 5I): "L'entrepreneur, quel qu'il soit, doit être considéré comme un être parasite vivant aux dépens de ceux qu'il exploite, car il ne fait rien par lui-même, il ne sert que de lien entre les travailleurs et les consommateurs; pour cela, il a la meilleure part du profit et il est maître commandant à ses ouvriers".

Il est curieux d'ailleurs de remarquer qu'en dépit de ces orientations nouvelles, Buchez conserve toujours, en partie, le vocabulaire saintsimonien. Nous l'avons vu ci-dessus employer l'expression "exploitation de l'homme par l'homme". C'est ainsi encore qu'un article du 23 juin I 832 s'intitule De la nécessité d'unir les Gouvernants, les Savants et les Industriels. Le 4 février, dans un article sur la Nationalité, on lisait: "Au milieu de toutes les variétés d'antagonisme ${ }^{1}$ de notre temps, il n'y en a qu'un qui soit puissant, qui ait plus de durée que celle d'une vie d'homme: c'est celui qui sépare les bourgeois des

1 Dans son Introduction à la Science de l'histoire (dont il va être question), p. 97, Buchez cite ce passage de l'opuscule de Kant Idee zu einer allgemeinen Geschichte in weltbürgerlicher Absicht: "Le moyen dont se sert la nature pour opérer le développement des dispositions de l'espèce, c'est l'antagonisme des hommes dans la société, comme pouvant y devenir enfin la source d'un ordre légitime", et il explique qu'il faut entendre par ce mot antagonistme "la lutte du bien contre le mal". Il ajoute que, traduit en français en $180 \mathrm{r}$, cet opuscule de Kant "doit être compté dans la somme des travaux qui ont servi de guide à l'esprit français modeme". Les saint-simoniens lui auraient-ils emprunté ce terme d'antagonisme, qui, à notre connaissance, ne se trouve qu'une fois chez Saint-Simon luimême? 
salariés". Le dernier numéro de cette série ${ }^{1}$ enfin contient cette phrase, qui rappelle la Doctrine: "La masse des hommes est liée au servage du salaire; elle n'est pas libre" et, résumant le programme qu'il s'était tracé, L'Européen conclut: "Nous avons vu la nation divisée en deux classes, les hommes de loisir et les hommes de travail; les égoïstes qui consomment et les dévoués qui produisent; les exploitants qui gouvernent et les salariés qui souffrent. Nous avons pensé qu'il fallait travailler à détruire cette opposition et non aider à la maintenir. Nous avons proposé les moyens de la faire disparaître successivement, en améliorant la condition des classes pauvres". Les "égoïstes" et les "dévoués", - "améliorer la condition" de la classe laborieuse, voilà encore des expressions toutes saint-simoniennes. Mais prenons bien garde que, sous ces expressions, Buchez met tout autre chose que ce qu'y avaient mis ses anciens amis: il s'agit pour lui désormais, grâce aux projets d'association ouvrière qu'il a exposés dans ces articles ${ }^{2}$, d'émanciper les travailleurs de leur condition de salariés, de les mettre en mesure d" "entreprendre eux-mêmes des travaux" et ainsi de "faire disparaittre" l'injuste opposition de la classe qui travaille et de celle qui possède les capitaux.

Au reste, quelques mois plus tard, dans son Introduction à la Science de l'histoire ${ }^{3}$, Buchez se montrera plus net et plus tranchant encore. On notera même dans sa critique une certaine âpreté, qui s'explique sans doute à la fois par les contacts ouvriers dont nous avons parlé, et par les constatations que lui avait permis de faire sa profession de médecin. C'était en effet l'époque où les conséquences de l'industrialisme naissant et de la libre concurrence retombaient de tout leur poids, sans qu'aucune loi sociale vînt les adoucir, sur la classe ouvrière. Les enquêtes d'un autre médecin. le docteur Villermé, et d'Emile Buret ${ }^{4}$ vont bientôt révéler la misère véritablement effroyable des ouvriers de cette époque. Buchez lui-même signale souvent le taux élevé de mortalité que les statistiques accusent chez les pauvres. Si noires que soient les couleurs sous lesquelles il nous dépeint la condition ouvrière de l'époque, on ne peut guère trouver ce tableau trop forcé. "Aujourd'hui, répète Buchez ${ }^{5}$, la société européenne est, sous le rapport des inté-

${ }_{1} 27$ octobre I832, p. 335. - Une nouvelle série de l'Européen parut d'octobre 1835 à octobre I 838 (Bibl. Nat.: R 6358-6359).

2 Cf. notre étude Buchez fondateur de l'Association ouvrière de production, dans le recueil déjà cité Hommes et Idéologies de 1840,1 .

3 Paris, Paulin, mai 1833.

- Villermé, Tableau de l'état physique et moral des ouvriers dans les manufactures de coton, de laine et de soie, I 840 (reproduction d'un rapport à l'Académie des Sciences Morales et Politiques); - Emile Buret, La Misère des Classes laborieuses en France et en Angleterre, 1841. - Pour l'Angleterre, bien des faits avaient déjà été signalés par Andrew URE, Philosophy of Manufactures, 1835.

${ }^{5}$ Introduction à la Science de l'histoire, p. 9. 
rêts matériels, partagée en deux classes... De ces deux classes, l'une est en possession de tous les instruments de travail, terres, usines, maisons, capitaux; l'autre n'a rien: elle travaille pour la première". Telle est du moins la situation dans ce que Buchez appelle les sociétés à base de chartes, qui sont fondées sur la propriété héréditaire. Cette hérédité entraîne celle des fonctions, de sorte que celles-ci se trouvent concentrées dans un assez petit nombre de familles qui, en fait, gouvernent. Qu'on n'objecte pas le partage égal des biens entre les enfants. D'abord, cette disposition ne peut aller contre cette loi générale, établie en économie politique, que, "sous notre régime industriel, les capitaux tendent à augmenter dans les mains où ils sont déjà accumulés en plus grandes masses et à échapper à ceux qui possèdent les richesses les plus faibles". Et, d'autre part, le seul effet de cette disposition a été "de porter des hommes qui seraient restés purement oisifs, à intervenir par leurs capitaux dans des opérations industrielles”. Or:

"La possession des instruments de travail serait, à elle seule, un avantage immense dans une société dont le travail serait l'unique loi. Mais aujourd'hui à cette possession est attaché le droit de lever sous le nom d'intérêts, de fermage, de loyers, un impôt sur le travailleur qui utilise l'instrument: et de là résulte la possibilité à des familles d'être héréditairement oisives, et héréditairement presqu'à l'abri de toutes les chances qui menacent plus ou moins tous les hommes qui se font chefs d'industrie.

"Nous appelons chefs d'industrie ces hommes qui, chargés par les riches du soin de faire fructifier leurs terres et leurs capitaux, moyennant le paiement d'un loyer, ou possesseurs d'une fortune trop faible pour vivre oisifs, se placent en tête du travail, spéculent et font des bénéfices pour leur compte, gouvernent immédiatement et paient les salariés et peuvent ainsi arriver, par la suite des générations, à se classer définitivement parmi les propriétaires oisifs".

Ces chefs d'industrie, "placés comme intermédiaires entre les oisifs heureux et les pauvres qui travaillent", se trouvent, d'après Buchez, dans une situation difficile. "En hostilité sourde avec les premiers, qui les rançonnent", ils sont "en guerre ouverte avec les seconds, qu'ils exploitent à leur tour". La seule loi de la vie économique actuelle étant la concurrence, il faut que le chef d'industrie vende le meilleur marché possible: “Il ne peut obtenir ce résultat qu'en diminuant le prix de la main d'oeuvre, et il y parvient par l'invention des machines et la baisse du salaire de ses ouvriers". Quelles sont les conséquences de cet état de choses pour les travailleurs? C'est ici que le ton de Buchez devient sombre et amer: 
"Le plus grand nombre des hommes naît au monde nu de tout héritage... Ceux-là n'ont guère le temps d'apprendre; ils n'ont pas le loisir du choix; presque dès leur premier jour il faut qu'ils vivent; ils sont destinés à exister dans une seule pensée, celle d'éviter la faim; attachés au sol comme des polypes, là où ils viennent au monde, ils travaillent et meurent; car, dans cette classe, les journaliers sont le bas peuple, et les ouvriers sont l'aristocratie. Si vous avez vécu avec eux, vous savez combien, dans les chaumières du pauvre campagnard, on élève haut le sort de celui qui possède, comme on dit, un métier.

"Les salariés sont les égaux des chefs d'industrie en ce sens que ceux-ci ne peuvent ni les tuer ni les battre ni leur refuser le salaire promis. Mais, du reste, ils sont complètement à leur disposition quant au taux de ce salaire et quant aux obligations qu'on leur impose; il leur est défendu de se coaliser ${ }^{1}$ pour se défendre et souvent, là même où la loi pourrait les protéger, ils sont liés par la faim à ne pas vouloir de son secours...

"L'invention des nouvelles machines et surtout les crises financières et les banqueroutes jettent annuellement une certaine masse d'hommes hors des fabriques où elle était employée et la forcent à chercher de nouvelles occupations. Ainsi, il se forme et se maintient une population ouvrière mobile sur place en quelque sorte et cherchant un salaire. Ces hommes n'ont point le temps d'attendre; lorsqu'ils manquent un jour de travail, c'est un jour de jeûne qu'il leur faut subir. Ils se donnent donc à quelque prix que ce soit, de sorte que ces malheureux luttent entre eux à qui sera employé; et leur moyen, c'est de s'offrir au plus bas prix possible".2

C'est ainsi qu'en Angleterre on a pu exiger des ouvriers jusqu'à seize heures de travail par jour. C'est ainsi que les enfants ont été utilisés pour certains travaux auxquels ils sont aussi aptes que les hommes ${ }^{3}$, et "leur application à ces oeuvres a été accueillie avec joie par les chefs d'industrie; car elle leur a donné le moyen de faire opérer ce travail à un très bas prix". Il arrive alors qu'en vue de cette utilisation les ouvriers cherchent à avoir beaucoup d'enfants et, chez "beaucoup"

1 On sait que les coalitions, c'est-à-dire les grèves, les ententes en vue de la cessation du travail étaient sévèrement punies par les articles 4 I 5 et 416 du Code pénal français, tandis que l'article 414, concernant les employeurs, ne punissait que les ententes tendant "à forcer injustement et abusivement l'abaissement des salaires". Ces dispositions ne seront abolies qu'en 1864 .

2 Ouv. cité, p. 23-25.

3 C'est seulement en i 84I que fut votéc en France la loi sur le travail des enfants dans les manufactures. 
d'entre eux, assure Buchez, "la paternité est devenue une spéculation". Recevant une nourriture insuffisante, exposés à tous les risques de maladies, ces enfants "seront chétifs et malingres; ils deviendront laids" et porteront sur leur face les stigmates de leur misère que l'on prendra pour ceux de la débauche: "Il est constaté qu'ils vivent plus de moitié moins que leurs maitres; ils mourront seuls, dans des hôpitaux, sans consolation, sans espoir". Et enfin, l'industrie utilisera les femmes: selon Buchez, dès cette époque, "les trois quarts au moins des femmes" sont des salariées. "Leur salaire est en général de moitié, quelquefois de deux tiers inférieur à celui d'un homme". Encore arrive-t-il, comme celui des hommes baisse sans cesse, qu'il descende au taux des salaires féminins, et alors ce sont les hommes qui prennent le travail des femmes. ${ }^{1}$

A cet état de choses, Buchez ne pense pas que l'aumône ou les oeuvres de charité soient un remède efficace. Tout au plus servent-elles "à amoindrir l'irritation de cette classe redoutable par son nombre et son désespoir", et faisant allusion sans doute aux poors laws, Buchez affirme même qu'elles "sont devenues dans ces derniers temps, particulièrement en Angleterre, un moyen de coërcition": car, pour en bénéficier, il faut avoir été "ouvrier honnête, c'est-à-dire soumis".

On voit que, chez Buchez, la critique saint-simonienne s'est infléchie en un sens nouveau: elle prend une allure revendicatrice qu'elle n'avait pas chez les auteurs de la Doctrine. On ne sera pas surpris de voir cette évolution se poursuivre et, en un sens, s'achever chez les rédacteurs de l'Atelier. Ce petit journal ${ }^{2}$ qui parut de 1840 à 1850 , était en effet rédigé exclusivement par des ouvriers disciples de Buchez. Nous aurons l'occasion de constater chez ceux-ci un accent tout particulier qui ne s'était pas laissé percevoir chez les auteurs précédemment étudiés, accent dû à leur expérience directe de la condition ouvrière, renforcée d'ailleurs par le fait qu'à la date où naît l'Atelier, les conflits du travail deviennent plus aigus: l'Atelier commence à paraître en septembre I840, au lendemain d'un mouvement de grèves qui avait affecté, à Paris, la presque totalité des corps de métiers.

Dès leur premier numéro, les "ateliéristes", comme on les appelait, exposent l'utilité d'un journal "adressé aux ouvriers par des ouvriers": "Jusqu' à ce jour écrivent-ils, les classes ouvrières ont été défendues par des gens qui leur étaient étrangers... Rien de pareil ne pourra être opposé à un journal tel que le nôtre".

Ce n'est pas qu'on ne retrouve encore sous leur plume des réminiscences du vocabulaire saint-simonien. Ils ne manquent pas de repren-

1 Ouv. cité, p. 26-28 et 31-33.

2 Cf. notre monogtaphie Un Journal d'Ouvriers: l'Atelier, Alcan, 1919; - 2me édition, Editions Ouvrières, Paris, 1954. 
dre la formule "exploitation de l'homme par l'homme". ${ }^{1}$ Mais ils précisent que le mot d'exploitation n'a rien d'hyperbolique ni d'analogique: il exprime "le fait exact, la vérité rigoureuse".2 En revanche, le terme d'industriels prend définitivement chez eux le sens que nous lui donnons aujourd'hui: un article de septembre i 847 oppose "nos seigneurs et maîtres, les industriels" à "nous, ouvriers". Il en est de même de l'opposition entre "les travailleurs" et "les oisifs" que l'on y rencontre surtout au début ${ }^{3}$ : "Tous les instruments de travail, expliquentils, sont la propriété exclusive d'une certaine classe qui acquiert par là le moyen de vivre dans l'oisiveté, ou au moins de bien vivre en travaillant très peu, tandis que l'ouvrier, en louant ses bras aux possesseurs, vit très mal en travaillant beaucoup et toujours". ${ }^{4}$

Parfois l'Atelier utilise aussi le langage fouriériste. C'est ainsi qu'il distingue dans la production trois éléments: le Capital, le Talent et le Travail. Mais on va voir qu'il apprécie leur rôle tout autrement que les disciples de Ch. Fourier. Sur le Capital, il est fort bref, mais péremptoire: c'est le Capital qui a "la haute main sur toutes choses, et tout fléchit devant lui". ${ }^{5}$ C'est surtout à propos du Talent, c'est-à-dire des chefs d'entreprise, des ingénieurs, etc, que se marquent ses divergences. La plupart des leaders de la doctrine phalanstérienne, - tel Victor Considérant, ce polytechnicien, - sont des représentants de cet élément social. Or, déclare sans ménagement l'A telier ${ }^{6}$, "nous sommes fatigués des exploitations de toutes sortes, et même, il faut le dire, de celle du Talent". Il ne nie certes pas que le "talent" doit être rétribué, mais seulement dans la mesure où il correspond à un travail réel. Or il n'en est pas ainsi. Le "talent" a partie liée avec le capital. Il participe largement à ce que l'Atelier appelle "le privilège industriel". Ici revient l'argument de Buchez: trop souvent, "le maitre, l'entrepreneur ou l'industriel, comme on voudra, ne connaît rien, ou à peu près, à la profession qu'il exploite; ses ouvriers et ses contremaîtres ou employés fonctionnent pour lui'. ${ }^{7}$ C'est comme participant à la propriété des "instruments de travail": terres, matières premières, outils, machines, capitaux, qu'il possède le privilège de "vivre du travail de ceux qui n'ont que leurs bras" et peut ainsi opérer sur la part du travailleur un prélèvement "inique et injuste" ${ }^{8}$ tout autant que celui du capitaliste proprement dit. Cette notion du "prélévement" sur le travail de l'ou-

1 Sept. 1840, p. 3 ; - avril I 842, p. 64.

2 Nov. I 846, p. 4 I I ; - cf. déc. I 849 , p. 444.

3 Par exemple, oct. I 840, p. 1 I.

4 Déc. 1840, p. 28.

5 Oct. 1846, p. 385 .

6 Mars I84I, p. 5 I.

7 Août r 84 I, p. 94.

${ }^{8}$ Déc. 1842, P. $30 ;-$ juill. 1843, p. 98. 
vrier que nous avions déjà rencontrée dans les doctrines précédentes, revient ici comme un leit-motiv: "impôt énorme", "dîme éternelle et écrasante", ce prélèvement est évalué par lui, en janvier I850, à un tiers environ de ce que produit l'ouvrier. Il s'est constitué ainsi, dit-il encore, une "nouvelle féodalité", une "féodalité industrielle" 1 , qui d'ailleurs a la loi pour elle, car "il n'y a point aujourd'hui de direction sociale: il n'y a que compression d'une classe par une autre". ${ }^{2}$ L'idée que le gouvernement et même la prétendue représentation nationale ne sont en réalité que les représentants " $d$ 'un seul intérêt, l'intérêt bourgeois" 3 revient souvent dans ses articles, du moins sous la Monarchie de Juillet: "Il serait parfaitement ridicule, écrit-il en octobre I 843 , d'attendre quelque chose d'une représentation exclusivement composée de hauts barons de l'industrie: une pareille assemblée ne représente et ne fait les affaires que de sa classe". 4

Même parmi ceux qu'on qualifie d'ouvriers, l'Atelier fait encore des distinctions, et il s'est attiré par là les critiques de journaux démocrates comme le National, voire de journaux soi-disant ouvriers. ${ }^{5}$ On parle, par exemple, d'"ouvriers patentés": ce sont, dit l'Atelier, des "ouvriersmaîtres" qui, ayant amassé un peu d'argent, se sont établis à leur compte et emploient à leur tour des salariés ${ }^{6}$ : "Ouvrier patenté est un nonsens; l'ouvrier qui paie patente est un entrepreneur; le véritable ouvrier est celui qui loue ses bras à un entrepreneur moyennant salaire" ": ces patentés sont le plus souvent les "maîtres" les plus durs. Il en est de même des contre-maîtres qui sont presque toujours, selon l'Atelier ${ }^{8}$, des "instruments d'oppression" et se montrent en général

1 L'Atelicr pub. toute une séric d'articles sous ce titre, de mars 1845 à novembre 1847 .

2 Juin $184 \mathrm{I}, \mathrm{p} .73$.

3 Juin 1847 , p. 525 .

4 Oct. 1843 , p. 2. - Les saint-simoniens avaient réservé au contraire des avantages considérables aux "comtes" et aux "barons de l'industrie".

3 Le National, 20 oct. 1840, lui reproche de prendre le mot "travailleurs" en un sens "beaucoup trop restreint". Le 6 oct. 1842, l'Artisan, moniteur ouvrier de Paris et des départements (Bibl. Nat.: Lc ${ }^{2}$ I 539 ) blâme "l'amertume et la vivacité de ses expressions" qu'il attribue au fait qu'il est exclusivement rédigé par des ouvriers. En mai I 844, dans l'Union, l'ouvrier saint-simonien Paton incrimine son dédain pour les institutions pacifiques (il s'agissait d'une caisse de retraites destinées à "cimenter l'union difficile, mais nécessaire des intétêts de la bourgeoisie et des intérêts du peuple").

${ }^{8}$ George Sand raconte dans la préface des Conteurs ouvriers (Paris, 1849 ) de l'ateliériste J. P. Gilland qu'on offrit un jour à celui-ci, ouvrier serrurier, les fonds nécessaires pour "s'établir": il refusa.

7 Mémoire sur la réforme de l'institution des prudhommes, publ. par l'Atelier, r843, p. 6.

${ }^{8}$ Fév. I 84I, p. 44. - Buchez au contraire avait considéré les contremaitres comme les représentants qualifiés des ouvriers. Dans l'Européen du 17 déc. 1831 , p. 39, il propose, pour les ouvriers d'usines, l'institution de "syndicats" obligatoires qui seraient organisés par le gouvernement et qui seraient chargés de répartir la main d'oeuvre, de fixer les salaires, etc. Or ces "syndicats" devtaient, selon lui, être composés "en partie de contremaîtres élus par leurs pairs, et en partie des délégués des fabricants". 
plus sévères pour l'ouvrier que les patrons eux-mêmes. En août I847, le journal La Presse ayant rapporté un meeting tenu à Manchester dans lequel les délégués des ouvriers employés dans les filatures avaient, d'une façon assez plate, exprimé leur respectueuse gratitude à "ceux qui leur donnent du travail" pour avoir réduit, en prévision d'une crise menaçante, leurs heures de travail plutôt que leurs salaires, l'Atelier ${ }^{1}$ demanda "à quelle classe de salariés" appartenaient ces délégués, - ce qui lui valut une lettre de Frédéric Engels, récemment revenu d'Angleterre, lui confirmant qu'il s'agissait en réalité d'un meeting de contremaîtres. L'Atelier ${ }^{2}$ s'empressa de publier la lettre d'Engels.

Restent les ouvriers proprement dits. L'Atelier dénonce d'abord leur profonde misère, et, comme il a été dit ci-dessus, il ne semble pas qu'à cette époque la réalité ait été plus brillante qu'il ne la dépeint. Au reste, il publie des tableaux comparatifs de salaires ${ }^{3}$, d'où il conclut que, de 1830 à 1848 , "le salaire a constamment baissé sur tous les points du territoire". ${ }^{4}$ Il rappelle d'ailleurs la loi que Lassalle nommera plus tard la "loi d'airain", mais qui, on le sait, avait déjà été énoncée par Ricardo: "C'est une loi dont l'empire est reconnu par tous les économistes que, dans tous les temps, le salaire est proportionné aux prix des denrées de première nécessité". Ainsi, "le paupérisme passe à l'état chronique" dans la classe ouvrière; c'est un mouvement irrésistible qui "pousse peu à peu le travailleur vers des régions de misère qui paraissent tout d'abord inaccessibles". ${ }^{5}$ L'Atelier insiste, lui aussi, sur la condition déplorable des enfants: n'a-t-il pas été révélé, lors de la discussion de la loi de I84I, que "de malheureux enfants de cinq à six ans travaillaient douze et quinze heures par jour dans des usines malsaines" 6 ? Quant aux femmes, l'Atelier signale surtout la misère de celles qui travaillent à domicile, plus exploitées encore que celles qui travaillent en usine, "ces pauvres femmes pâles, défaites, exténuées par un travail de seize ou dix-huit heures et de trop longues veillées dans des taudis mal clos".7

Mais ce n'est pas seulement la misère ouvrière qui révolte l'A telier. Il y a, dans ses critiques, un autre thème et un autre ton: "Nous parlons

1 Les maîtres et les ouvriers en Angleterre, oct. 1847 , p. 10.

2 Même titre, nov. I 847, p. 24-25. - Nous avons publié la lettre d'Engels dans Hommes ct Idéologies de I $840, \mathrm{~V}, \S \mathrm{F}$.

3 Notamment déc. 1840 , p. 29 ; - mai 1841 , p. 70 ; - oct. 1848 , p. 223 , etc. - on trouve également dans ses colonnes des enquêtes sur le niveau de vie des différentes professions ouvrières.

1 Juin 1850, p. 547 .

5 Fév. I 846, p. 259 ; - août I 849 , p. 555 .

- Janv. I84I, p. 36 .

7 Juin 184 I p. 77 ; - déc. 1842 , p. 31 . 
ici d'affranchissement, écrit-il en mars 1844 , et, quoiqu'il s'agisse de l'ordre économique, nous ne plaidons pas la cause des appétits, mais celle de la dignité et de la liberté des travailleurs". Nous faisions allusion plus haut à "l'expérience" de la condition ouvrière dont témoignent ses articles. Voici un de ces témoignages: "Il faut avoir été ouvrier soi-même et comme tel s'être rendu compte de toutes les blessures faites à la dignité du travailleur!' Blessures, ajoute-t-il, qu'on inflige parfois sans le vouloir, par habitude du commandement. Mais "les inférieurs ne les ressentent pas moins vivement; ils sont las de dévorer l'humiliation de leur état d'instruments; ils ont d'ailleurs mesuré la supériorité de leurs maîtres, et ils l'ont considérée comme un pur et simple préjugé". 1 Voilà, n'est-il pas vrai? un "son de cloche" que nous n'avions pas entendu jusqu'ici. L'Atelier n'a pas assez de sarcasmes contre ceux qui ne connaissent que "l'atelier et le ratelier", comme il dit un jour ${ }^{2}$ dans une polémique avec le journal des ouvriers saintsimoniens, la Ruche populaire. Il ne s'agit pas tant, à ses yeux, d'une question de bien-être que d'une question de dignité. "Tout souffrir, tout sacrifier, proclame-t-il ${ }^{3}$, plutôt que de subir la blessante dépendance dans laquelle on prétend maintenir notre classe". Un certain orgueil ouvrier n'est même pas absent de ses revendications: l'ouvrier, répète-t-il sans cesse, est "le vrai producteur", "le producteur réel" 4 , et cette classe que "le beau monde" appelle avec mépris la "classe inférieure", c'est "celle qui défend la patrie ${ }^{5}$, qui récolte le pain pour tous, qui construit les villes, qui fait tout enfin". ${ }^{6}$ Voilà pourquoi il s'indigne de ce "prélèvement" dont nous parlions ci-dessus. Demandez, dit-il, à l'ouvrier des villes ou des campagnes la raison de son mécontentement: "Je me plains, répondra-t-il, des prélèvements de toute nature qui sont faits sur le produit de mes sueurs. Voilà ce qui blesse cruellement et mon intérêt et ma dignité".?

Cette analyse s'accompagne enfin, chez les rédacteurs de l'Atelier d'un sentiment très aigu de l'opposition des classes: "La lutte, l'antagonisme ne cesse pas un moment entre le maître et l'ouvrier; c'est cette lutte, toujours sourde quoique douloureuse, qui se révèle parfois au monde sous les noms d'interdiction, de mise-bas, de coalition et de

1 Déc. 1849, p. 445 .

2 Nov. I 843 , p. I9.

3 Nov. 1845 , p. 224 (à propos de la mort d'un de ses rédacteurs, dont il dit: "Personne plus que lui ne fut jaloux de la dignité de l'ouvrier").

4 Notamment nov. I 840 ; août I 841 ; avril I 844 ; juill. 1845 ; nov. I 846 ; etc.

5 On sait que, par le procédé du "remplacement", les jeunes gens aisés pouvaient alors se dispenser du service militaire.

${ }^{6}$ Nov. $x 846$, p. 413 .

7 Juill. I 850, P. 567. 
rassemblement ${ }^{1}$, et qui se fait continuellement sentir dans chaque atelier". "La coalition est, dit-il ailleurs ${ }^{3}$, une des formes les plus fréquentes de la lutte permanente qui existe entre les intérêts des maîtres et des ouvriers". Et qu'on ne se fasse pas illusion: "Tant qu'il y aura des maîtres et des ouvriers, on pourra régler la lutte, la rendre légale et pacifique, mais enfin il y aura toujours lutte". ${ }^{4}$ Certains parlent de solidarité entre employeurs et employés. Tel n'est pas l'avis de l'Atelier: "Les maitres, il est clair que leur intérêt est directement opposé à celui des ouvriers". 5

Ce vif sentiment de l'opposition des classes eut l'occasion de s'affirmer à propos de L'article de La Presse dont il a déjà été question et qui motiva sa correspondance - toute occasionnelle d'ailleurs - avec Engels. La Presse s'était appuyée sur le meeting de Manchester pour célébrer l'harmonie du capital et du travail, "ces deux frères jumeaux de l'industrie". Écoutons comment, avant même d'avoir reçu la lettre d'Engels, l'Atelier réagit:

"Ah, pour le coup, ceci est trop fort... N'est-ce pas le système politique que préconise la Presse qui attribue au capital tous les bénéfices de l'entreprise et ne laisse en partage aux travailleurs que les privations ou la misère? Qu'y a-t-il au fond de ces creuses théories économiques si ce n'est l'exploitation du salaire? Vous qui écrivez que le bien-être de celui qui loue ses bras ne peut s'acquérir par la ruine de celui qui l'emploie; que les mauvais jours de l'un ne seront jamais les bons jours de l'autre, prétendriez-vous qu'il y a association entre le capitaliste et le salarié? Ne savez-vous pas que les mauvais jours de l'ouvrier sont les bons jours du maitre? que plus il y a de malheureux sans pain, plus les bras sont à meilleur marché et que la misère du pauvre fait la richesse du propriétaire? L'offre et la demande, n'est-ce donc plus la base de votre système économique?"6

Aussi l'Atelier n'entrevoit-il de solution au problème des classes que dans une transformation radicale du régime économique: "Il faut que le titre du travailleur change, que d'instrument il devienne homme".?

1 C'étaient les divers noms qu'on donnait alors à la "grève": l'ancien article 416 du Code pénal punissait les ouvriers "qui auront prononcé des amendes, des défenses, des interdictions...".

2 Juill. 1842, p. 87 .

3 Janv. 1842 , p. 35 . - C'est sous le titre "lutte du travail contre lc capital" que l'Atelier rend compte des grèves (sept. et nov. I 844 ; juill. 1845 ).

4 Juill. 1842, p. 87 .

5 Fév. I $84 \mathrm{I}$, p. 44 .

6 Oct. I 847, p. I I.

7 Déc. 1849, p. 445 . 
Encore cette transformation devra-t-elle s'accomplir surtout par "la volonté des intéressés". Il est vain d'attendre des privilégiés de l'ordre actuel une nouvelle nuit du 4 août, il est vain de compter sur cette "décevante providence" 1 qu'on nomme l'Etat: "Les améliorations dans la condition du peuple ne peuvent être que l'ouvrage du peuple lui-même".2

Avec cette phrase, nous aboutissons à une formule qui fait songer à la phrase du Manifeste communiste à laquelle nous avons déjà fait allusion: "L'émancipation des travailleurs sera l'oeuvre des travailleurs eux-mêmes". Cette analogie ne doit cependant pas faire illusion. Sous la ressemblance des formules, des inspirations très différentes se cachent. Albert Thomas le dira encore des ouvriers du Second Empire: "C'est par des sentiments de dignité, de fierté plus encore peut-être que par des sentiments d'opposition et de lutte que s'exprime alors la conscience de classe du prolétaire parisien". ${ }^{3}$ A plus forte raison est-ce vrai des ouvriers de l'Atelier et, plus généralement, des ouvriers de I 848. Ce qui les inspire, c'est un idéal de démocratie sociale. Le "privilège industriel" leur paraît être de même nature que le privilège politique: "La chose politique et la chose économique ne sont que les moyens d'un même but". 4 Il s'agit d' "élever les classes infériorisées au niveau des classes privilégiées", de réaliser "la république dans l'atelier", et l'association ouvrière de production leur paraît le moyen le plus sûr de cette transformation.

Ces différences une fois marquées, il suffit cependant de jeter un coup d'oeil en arrière sur l'évolution que nous avons essayé de retracer, pour mesurer le chemin parcouru depuis Saint-Simon. Les changements de sens subis par des expressions telles que "industriels", "travailleurs", "antagonisme", jalonnent les étapes de cette évolution qui arrive à maturité chez les rédacteurs de l'Atelier. Chez eux, la "conscience de classe" est déjà très éveillée et la notion, semée par SaintSimon, du "véritable producteur" prend un sens extrêmement précis. En somme, ils sont parvenus, avant les écrits de Marx et indépendamment d'eux, à une idée très nette, très rigoureuse même, de la classe sociale, définie moins par le niveau de vie que par les rapports de production et la place du travailleur dans le système de ces rapports. On a souvent remarqué que Marx lui-même, dans le Manifeste de I 848, utilise le mot classe de façon fort imprécise et qu'en déclarant que l'histoire des sociétés humaines jusqu'à ce jour se ramène à celle

\footnotetext{
1 Juill. I 850 , p. 570 .

2 Sept. I 842 , p. I.

3 Histoire Socialiste, t. X: le Second Empire, p. 185.

4 Oct. 1843 , p. 2.
} 
des antagonismes de classes, il cite comme exemples les oppositions entre hommes libres et esclaves, patriciens et plébéiens, seigneurs et serfs, maîtres-artisans et compagnons et, plus généralement, entre "oppresseurs" et "opprimés", parallèlement à celle entre bourgeois et prolétaires, et qu'il mêle ainsi "castes, ordres féodaux, hiérarchies de la corporation médiévale" avec les classes proprement dites. ${ }^{1}$ Il n'a d'ailleurs jamais mis au point de définition plus élaborée, et l'on sait que le Capital se termine sur un développement inachevé où il semble d'abord distinguer non pas deux, mais trois classes caractérisées par "l'identité des revenus et leurs sources": salaire, profit et rente, de leurs membres. ${ }^{2}$ Guidés par par leur expérience de la condition prolétarienne, les simples ouvriers de l'Atelier, en dépit ou peut-être à cause même de leur différence de point de vue avec le marxisme qui leur était encore inconnu, ont abouti à une notion plus consistante des classes sociales et de leur opposition. On sent déjà s'annoncer chez eux cette lutte que, dès avant I 848 et non sans effroi, pressentait Tocqueville, entre "deux classes: les bourgeois et le peuple dressés les uns contre les autres". ${ }^{3}$

1 P. Sorokin, Society, Culture and Personality, Harper and brothers, Londres et New York 1947, Part four, Chapt. I4, \$ III, p. 266-267.

2 Le Capital, trad. fr. Borchardt et Vanderrydt, Giard et Brière, Paris, I9O2, livre III, tome 2, p. 494 .

${ }^{3}$ Souvenirs, Paris, I893, cité par Maxime Leroy, Histoirc des idées sociales en France, Gallimard, Paris, 1954, tome III, p. 62. 